\title{
STEM-EELS Investigation of Charge and Strain Distributions in Perovskite Oxide Thin Films
}

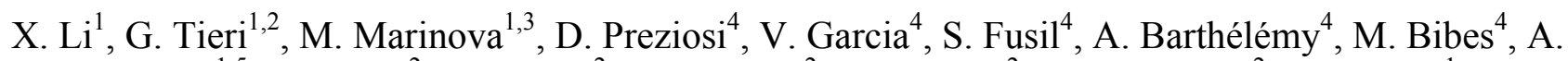
Torres-Pardo ${ }^{1,5}$, J. Fowlie ${ }^{2}$, M. Gibert ${ }^{2}$, S. Catalano ${ }^{2}$, S. Gariglio ${ }^{2}$, J.M. Triscone ${ }^{2}$, A. Zobelli ${ }^{1}$, M. Tencé $^{1}$, L. Bocher ${ }^{1}$, O. Stéphan ${ }^{1}$, C. Colliex ${ }^{1}$, A. Gloter ${ }^{1}$

${ }^{1 .}$ Laboratoire de Physique des Solides, CNRS UMR 8502, Université Paris-Sud, Orsay, France.

2. DQMP, Université de Genève, 24 Quai E.-Ansermet, Geneva, Switzerland.

3. now at, Institut Eugène Chevreul - Université Lille1, Villeneuve d'Ascq, France.

4. Unité Mixte de Physique, CNRS, Thales, Univ. Paris-Sud, Université Paris-Saclay, Palaiseau, France.

${ }^{5}$ now at, Department of Inorganic Chemistry, Complutense University of Madrid, Madrid, Spain.

Metal oxides and particularly their nanostructures have emerged as an important class of materials with a rich spectrum of properties and a great potential for device applications. Various interesting properties like exotic metal-insulator transitions, magnetoresistance, ferroelectricity have been reported for $\mathrm{ABO}_{3}$ perovskite oxides. They implicate interplays between structural, charge, spin and orbital degrees of freedom. Furthermore, new properties can be obtained for thin films due to strain, interface discontinuity, confinement, etc...

We will present how EELS techniques can nowadays quantify the amount of charge at the transition metal site with an atomic resolution. In the same time, structural degrees of freedom such as cationic displacement or oxygen rotation can be quantified by combining several STEM imaging modes.

First examples concern the investigation of the origin of magnetism and transport properties in nickelate and manganite thin films, including their bi-layers [1] and superlattices [2]. The $\mathrm{LaMnO}_{3} / \mathrm{LaNiO}_{3}$ (LMO/LNO) heterostructures display an intrinsic interface structural asymmetry depending on the growth sequence (LMO-on-LNO interface is sharper than the LNO-on-LMO one). Using a variety of spectroscopy-based techniques, we show that the degree of intermixing at the monolayer scale allows interface-driven properties such as charge transfer (figure 1) and the magnetic moment in the nickelate layer to be controlled [1].

Second series of examples concern the electric field control of functional properties in oxide-based electronics. Recently, ferroelectric $\mathrm{BiFeO}_{3}(\mathrm{BFO})$ has been used to provide a ferroelectric gating in perovskite heterostructures combined with a doped Mott insulator channel (Ce doped $\left.\mathrm{CaMnO}_{3}, \mathrm{CCMO}\right)$ [3]. These physical properties strongly depend on the strain and charge distribution in these devices. In this work, we report that charge distribution next to the BFO/CMO interface is highly inhomogeneous by combining grazing-incidence hard $\mathrm{x}$-ray photoemission spectroscopy and STEM-EELS measurements [4]. Typical sheet carrier densities in the range of $10^{14} \mathrm{~cm}^{-2}$ are obtained by EELS that are in line with Hall effect measurements but lower than expected to screen the BFO ferroelectric polarization. Ab-initio modeling concludes of the crucial role of plane termination [4]. The structure of ferroelectric domains in the BFO thin films will also be discussed with respect to the charge in the CMO channel. In particular, BFO structures for different ferroelectric polarization reversed by applying an electric field before the sample preparation for STEM observation, will be discussed (figure 2). 
References:

[1] M. Gibert et al, Nano Letters 15 (2015), p. 7355.

[2] M. Gibert et al, Nature Communications 7 (2016), p. 11227.

[3] H. Yamada et al, Scientific Reports 3 (2013), p. 2834.

[4] M. Marinova et al, Nano Letters 15 (2015), p. 2533.
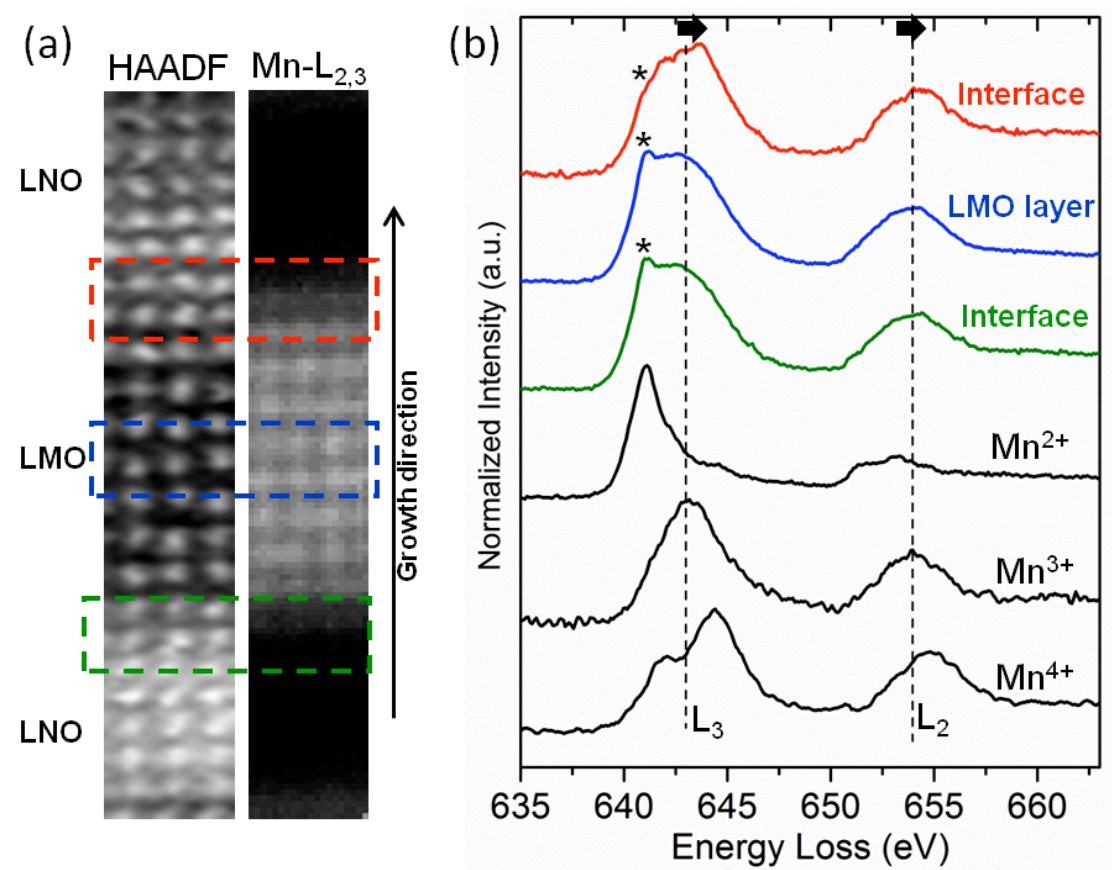

Figure 1. (a) HAADF image and $M n-\mathrm{L}_{2,3}$ chemical map corresponding to the superlattices (8u.c.LNO/8u.c.LMO)//(001)STO (b) EELS spectra of the $\mathrm{Mn} \mathrm{L}_{2,3}$-edges at the rough interface (red), at the inner LMO layer (blue) and at the sharp interface (green). Reference $\mathrm{Mn} \mathrm{L}_{2,3}$-edges for $\mathrm{Mn}^{2+}, \mathrm{Mn}^{3+}$ and $\mathrm{Mn}^{4+}$ are added for comparison (black). Notice the higher energy of the Mn- $\mathrm{L}_{2,3}$ edge at the rough interface showing $\mathrm{Mn}^{4+}$ valence.
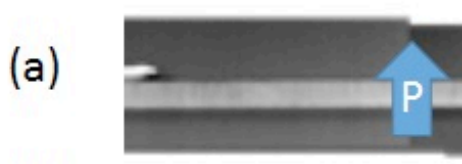

(b)
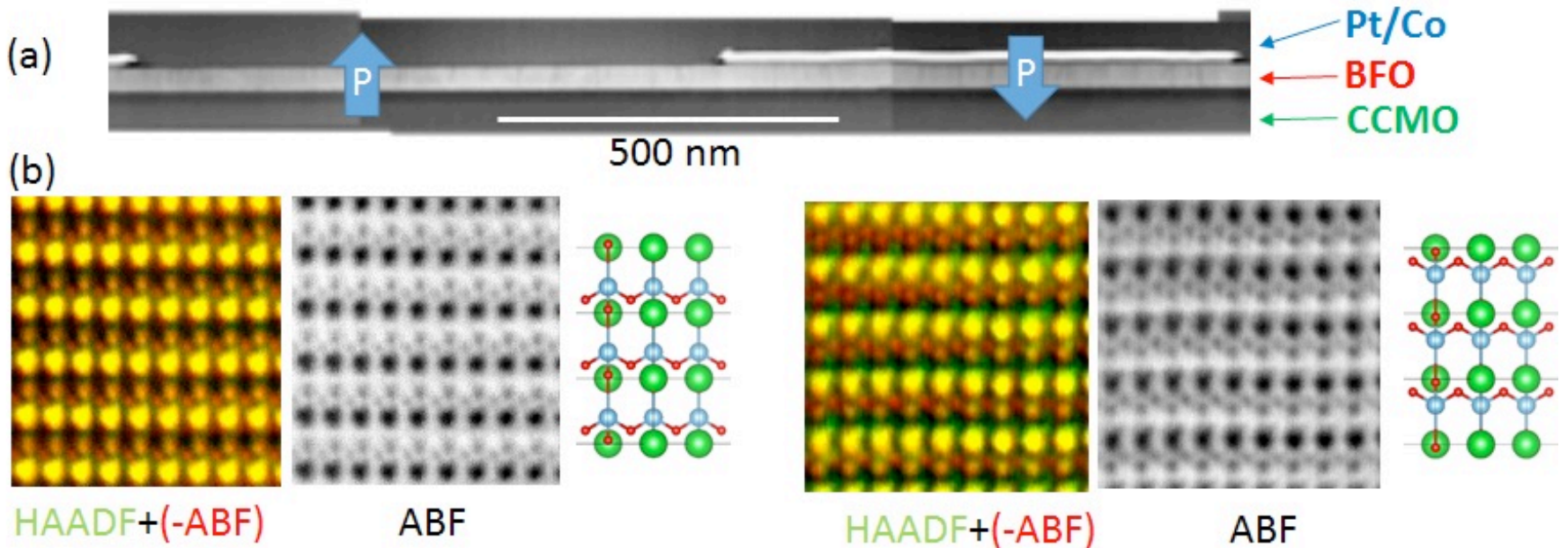

Figure 2. (a) low magnification HAADF-STEM images of BFO film grown on CCMO film. (b) ABF and composite (HAADF-ABF) STEM images from BFO domains with ferroelectric polarisation polled upward (left) and downward (right). 